\title{
QUERER Y PODER: (DES)IGUALDAD EN LA UNIVERSIDAD PÚBLICA ESPAÑOLA ${ }^{1}$
}

\author{
Sara Martín Bardera \\ Universidad de Salamanca
}

\begin{abstract}
RESUMEN: En este artículo se analizan, en primer lugar, los desequilibrios de género en la universidad pública española como un reflejo de la desigualdad social. En este sentido, se presentan datos sobre la comunidad universitaria para, a partir de ahí y en el marco de los cambios legislativos habidos en España desde la década del 2000, revisar en segundo lugar una serie de acciones vinculadas a la implementación de las políticas de igualdad de género. La existencia de unidades y planes de igualdad, o la formación de los equipos de gobierno atendiendo al principio de presencia equilibrada, son medidas que no tienen un coste elevado cuando, además, hay capital intelectual. Se concluye que el resultado de este análisis apuntará a la existencia o no de voluntad institucional para materializar el compromiso con la igualdad entre mujeres y hombres y se hacen algunas propuestas para mejorar la situación.
\end{abstract}

PALABRAS CLAVE: Políticas de igualdad de género, universidad pública española, unidades de igualdad, empoderamiento de las mujeres.

\section{WE WANT BUT WE CAN'T: GENDER (IN)EQUALITY IN THE SPANISH PUBLIC UNIVERSITIES}

\footnotetext{
ABSTRACT: First, this paper analyzes the gender gaps in the Spanish public universities as a reflection of social inequality, with data on the university community. Second, within the changes in the Spanish laws since the first decade of the $21^{\text {st }}$ century, we propose the evaluation of a series of measures associated with gender equality policies. Gender equality plans and gender equality units, or the formation of governing teams following a balanced presence criterion don't have a high cost for universities and public

1. Este artículo se publica en el marco del proyecto de investigación: "Bioética y ciudadanía (V): Deliberación estructurada: Análisis bioético en marcos institucionales" (FFI2015-63500-R) financiado por el Ministerio de Economía y Competitividad y dirigido por la Prof. Dra. M. ${ }^{a}$ Teresa López de la Vieja, a quien agradezco además las sugerencias realizadas para la elaboración del mismo.
} 
administrations when there is intellectual capital as well. The result of this analysis will point the existence (or not) of institutional will to materialize the compromise to reach equality between women and men.

KEYWORDS: Gender Equality Policies, Spanish public universities, equality units, women's empowerment.

Recibido: 30/03/2017

Aceptado: 19/06/2017

Correspondencia: Sara Martín Bardera, Universidad de Salamanca, Facultad de Farmacia Campus Unamuno, 37007 Salamanca. Email: bardera@usal.es.

\section{INTRODUCCIÓN}

En este artículo se toma como referencia el desarrollo de las políticas de igualdad en España, a partir de la década de 2000, para revisar la situación actual de las mujeres en la universidad pública española. La hipótesis de trabajo es que la crisis no influye en la implementación de algunas medidas a las que obliga la ley de igualdad y que cuando no se adoptan o es escaso su desarrollo se debe básicamente a falta de voluntad institucional.

En un primer momento, los datos reflejan que no hay discriminación directa, sino que las desigualdades y sesgos de género reflejan la desigualdad social. La persistencia de estos sesgos entre titulaciones y categorías académicas, la falta de correspondencia entre el número de mujeres que estudian y sus resultados académicos respecto a su desarrollo como docentes e investigadoras, tienen que ver, sobre todo, con la existencia de un techo de cristal. Si bien la universidad, en el conjunto del sistema educativo, ha proporcionado herramientas conceptuales y una formación especializada que ha permitido a las mujeres posicionarse mejor profesionalmente, ello no ha sido suficiente para paliar los efectos de una socialización de género, construida a partir de dos modelos sexuales hegemónicos.

Es aquí donde se espera que la universidad pública, como parte de la administración del estado e institución de enseñanza, contribuya a erradicar las desigualdades. La LOI contempla dos herramientas fundamentales para ello: las unidades (UI) y los planes de igualdad (PI). Por ello, en un segundo momento, se analiza cuál ha sido la respuesta de las universidades: si hay unidades y planes, cómo se conforman y su continuidad. En este artículo se defiende que la autonomía universitaria y su capital intelectual son puntos fuertes para desarrollar políticas de igualdad, y que la crisis económica no es razón suficiente para no implementar las medidas que aquí se revisan.

\section{Marco Legislativo}

La educación y la enseñanza como instrumentos para promover el respeto a los derechos y libertades fundamentales, sin distinción por razón de sexo, están presentes en el ordenamiento internacional que establece Naciones Unidas desde 1945. Las sucesivas 
declaraciones sobre los derechos de la mujer, las cuatro Conferencias dedicadas a ella y los Objetivos de Desarrollo del Milenio, han ido extendiendo la idea de que la emancipación de la mujer está necesariamente vinculada a su capacitación. En la Unión Europea (UE), donde en la actualidad la presencia de la mujer es equilibrada en todos los niveles educativos, el enfoque sobre las desigualdades se centra más en la necesidad de erradicar la persistencia de estereotipos que influyen en la elección de titulaciones, o en la baja presencia de mujeres en la investigación y en las categorías profesionales más altas.

En España, las políticas de igualdad que se iniciaron con la Transición democrática², alcanzaron su mayor desarrollo legislativo e institucional durante la primera década del $2000^{3}$. En esta etapa son clave ${ }^{4}$ dos leyes orgánicas: la 1/2004, de 28 de diciembre de Medidas de protección integral contra la violencia de género (LVG) y la 3/2007, de 22 de marzo, para la igualdad efectiva de mujeres y hombres (LOI). En la línea marcada por la UE, la LOI establece que para integrar el principio de igualdad en las políticas educativas hay que eliminar prejuicios sexistas presentes en los estereotipos de género, así como promover la presencia de mujeres en los órganos de gobierno ${ }^{5}$.

Los postulados de la LOI para la educación superior ${ }^{6}$ se reflejan en la Ley Orgánica 4/2007 de 12 de abril (LOU4) ${ }^{7}$ cuando establece que la universidad, como trasmisora de valores, debe incorporar el respeto a la igualdad y procurar mecanismos para alcanzar la paridad en los órganos de representación y gobierno, promoviendo la participación de las mujeres en los equipos de investigación, además de elaborar programas sobre igualdad de género. Resalta así su papel proactivo en la consecución de la igualdad material, para lo que serán clave unidades y planes de igualdad.

Este marco legislativo responde al desarrollo del concepto de género en el pensamiento feminista y, sobre todo, a su traslado en la generación de políticas públicas. Un traslado que significa asumir que la desigualdad entre mujeres y hombres es estructural y, al tiempo, un problema de justicia ${ }^{8}$. Para llegar a este significado de la desigualdad como desigualdad de género, debe tenerse en cuenta el marco teórico que se establece a partir del feminismo ilustrado y el feminismo de la igualdad en el debate con el feminismo de la diferencia ${ }^{9}$. Ello ayuda a comprender cómo los patro-

2. Sobre la influencia internacional y el menor peso de los movimientos feministas en los cambios institucionales de esta época, véase Astelarra (2005: 137-144) y Martínez (2014: 158).

3. Durante el gobierno del socialista José Luis Rodríguez Zapatero (2004-2011, VIII y IX legislaturas), el desarrollo legislativo de la igualdad y la creación del Ministerio de Igualdad (2008-2010) son la base de lo que Lombardo y León (2014: 16-23) denominan régimen de género.

4. Son clave por su carácter orgánico, por la asunción del concepto de género con el significado del que le dota el feminismo teórico, así como por incorporar el gender mainstreaming y las acciones positivas.

5. Por eso es importante que se incluya el principio de igualdad en los currículos, la formación del profesorado y los proyectos; además de enseñar el papel de la mujer en la Historia (LOI, 2007: art. 24).

6. LOI, art. 25.

7. Modifica la Ley Orgánica 6/2001, de 21 de diciembre, de Universidades.

8. Sobre el marco teórico que encuadra este estudio, véase Martín Bardera (2016) donde se analiza lo que ha significado trasladar el complejo concepto de género, como categoría de análisis, desde las teorías feministas a las políticas públicas. Aquí se defiende que es la primera vez que las demandas feministas entran en la agenda política como un proyecto integral que crea un nuevo paradigma, destacándose la perspectiva y la transversalidad de género como claves para orientar la consecución de la igualdad efectiva a partir de un doble movimiento de corrección y promoción.

9. Martín Bardera (2016: 290-293; "Referencias bibliográficas" 207-311). 
nes de género orientan a los individuos, desde los distintos procesos de socialización, a perfilar sus opciones y prioridades en función de dos modelos hegemónicos, según sean hombres o mujeres. Es algo que explica no sólo el sesgo en la elección, sino los techos de cristal en el desarrollo profesional, como se verá más adelante.

A continuación se ofrecen datos para valorar la situación de las mujeres en los tres colectivos de la universidad pública española: Personal Docente e Investigador (PDI), Personal de Administración y Servicios (PAS) y Colectivo Estudiantil (CE) (Tabla 1).

\section{COMUNIDAD UNIVERSITARIA EN FEMENINO PLURAL}

La distribución de mujeres por ramas de estudio y por categorías profesionales permite comprobar, en la misma institución, los desequilibrios entre formación y empleo.

\section{Algunos datos}

Los datos sobre las mujeres en el PDI son relevantes, porque en esta etapa culmina la de estudiante. La existencia de otro colectivo profesional, el PAS, permite ahondar en el significado de las diferencias en el ámbito laboral, como se explicará.

La situación es de sobra conocida: mayor acceso de las mujeres a la universidad, mejor rendimiento como estudiantes y menor presencia entre el PDI (Tabla 1). Durante la etapa de formación el sesgo de género está en la elección de titulaciones, siendo la diferencia más acusada en las ramas de Ingeniería y Arquitectura y de Ciencias (CC.) de la Salud (25,9\% y 69,7\% de mujeres, respectivamente). Sólo hay equilibrio de género en Ciencias (que agrupa las titulaciones menos cursadas), pues en Humanidades y CC. Sociales y Jurídicas, son mayoría las mujeres ${ }^{10}$. En el paso de máster a doctorado se pierde capital femenino: el 56,2\% de mujeres, sobre el total de egresados en máster, baja al 49,3\% en tesis defendidas. El punto de inflexión se halla en el subprograma estatal de incorporación ${ }^{11}$, donde no llegan al 40\% en las ayudas Ramón y Cajal (35,9\%) y están en el $46,2 \%$ en las de formación postdoctoral ${ }^{12}$. Si bien en el mismo programa las contratadas como técnicos de apoyo se acercan al 60\%, aquí la titulación máxima exigida es graduado superior ${ }^{13}$ y es la categoría menos dotada económicamente ${ }^{14}$.

Las mujeres en el PDI (Tabla 1) representan el 39,4\% y ocupan mayormente las categorías profesionales inferiores, esto es, las que requieren menos acreditación, tienen peores sueldos y menor oportunidad de promoción. Si se diferencia entre personal funcionario y laboral, las funcionarias disminuyen al 35,4\% con relación al total de funcionarios PDI; mientras las contratadas pasan a ser el $43 \%{ }^{15}$. Por categorías, las catedráticas de universidad (CU) alcanzan por primera vez el 20\% en el curso 2012-2013 y las titulares universitarias (TU) se aproximan al $40 \%$ en el

10. MECD: EPU, curso 2013-2014.

11. Plan Estatal de investigación científica y técnica y de innovación (PECTI).

12. En el informe anterior, las mujeres RyC, Juan de la Cierva y técnicos de apoyo eran el $39 \%$, el $38 \%$ y el $57,5 \%$ respectivamente (datos respecto al total de ayudas concedidas a la universidad. MECD, 2014).

13. Subprograma Personal Técnico de Apoyo (PTA) Ministerio de Ciencia e Innovación (MICINN, 2012).

14. Las más dotadas fueron RyC: 51.536.200 € para 167 ayudas y, las menos, las de técnicos de apoyo: $6.480 .000 €$ para 180 (MECD: 2015, 151).

15. 47.075 funcionarios, 46.642 contratados y 635 eméritos (MECD: EPU, curso 2013-2014). 
Tabla 1. Mujeres en la comunidad universitaria. Curso 2013-2014

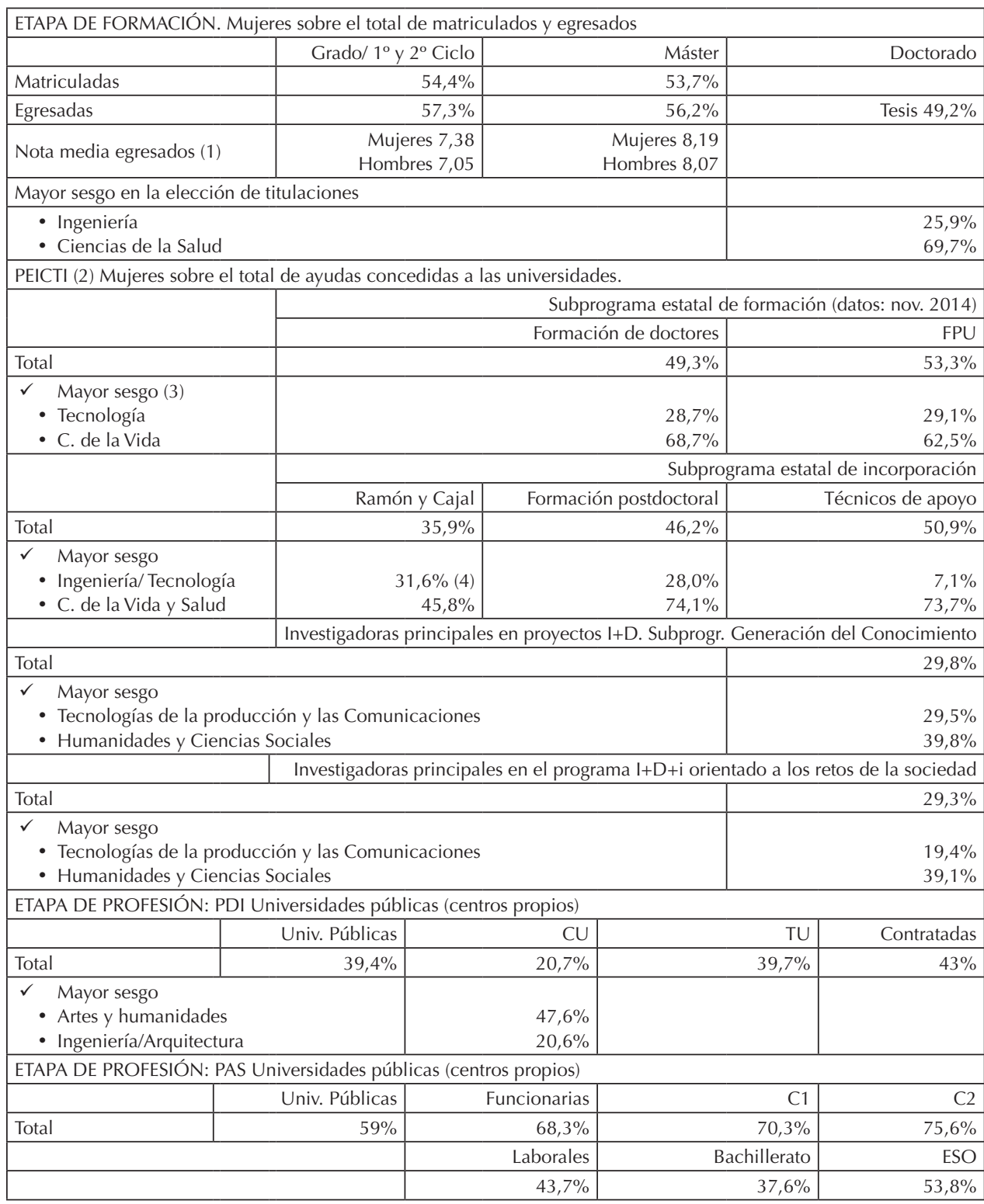

(1) Las tasas de rendimiento, éxito y evaluación no vienen desagregadas por sexo. En 2011, la tasa de rendimiento de las mujeres estaba 10 pp por encima de los varones. Ministerio de Educación (MECD, 2011: 13) INE (2012: 4).

(2) Plan Estatal de investigación científica y técnica y de innovación. I+D+I. (MECD: 2015, 145-157).

(3) Las áreas temáticas en el caso de la formación de doctores se denominan "Tecnologías de la producción y las comunicaciones" y "Ciencias de la Vida y Agroalimentación", respectivamente.

(4) Aquí, el porcentaje de mujeres más bajo sobre el total, está en Ciencias Básicas: 26,9\%.

Fuente: Elaboración propia a partir de los datos del MECD (2015) y de la Estadística de personal de las universidades (EPU), Curso 2013-2014. 
2013-2014. En diez años ${ }^{16}$ las CU aumentan 6,8 pp y 3,4 pp las TU (en conjunto el incremento es de 2 pp), pasando las contratadas del $37 \%$ al $43 \%$ (Tabla 2).

Tabla 2. Mujeres en el PDI. 2004-2013

\begin{tabular}{|c|c|c|c|c|c|c|c|c|c|c|}
\hline$\%$ PDI & CURSOS & ACADÉMI & $\cos$ & & & & & & & \\
\hline Categorías (1) & 2004-05 & 2005-06 & 2006-07 & 2007-08 & 2008-09 & $2009-10$ & 2010-11 & 2011-12 & 2012-13 & 2013-14 \\
\hline $\mathrm{CU}$ & 13,86 & 14 & 14,29 & 14,99 & 15,25 & 16,8 & 18,38 & 19,53 & 20,29 & 20,66 \\
\hline TU & 36,33 & 36,38 & 36,59 & 37,1 & 37,28 & 38,12 & 38,92 & 39,34 & 39,68 & 39,72 \\
\hline CEU & 33,48 & 32,74 & 32,92 & 31,73 & 30,57 & 30,16 & 29,39 & 29,19 & 29,21 & 29,28 \\
\hline TEU & 41,34 & 41,27 & 41,39 & 41,39 & 41,26 & 41,58 & 41,4 & 41,1 & 40,89 & 41,14 \\
\hline $\begin{array}{l}\text { Otro p. } \\
\text { funcionario }\end{array}$ & 26,12 & 31,01 & 22,86 & 28,44 & 34,38 & 35,47 & 30,89 & 32,11 & 34,36 & 37,50 \\
\hline Funcionaria(2) & 33,5 & 33,5 & 33,7 & 33,9 & 33,78 & 34,31 & 34,73 & 34,98 & 35,20 & 35,37 \\
\hline Contratada (3) & 37,23 & 37,49 & 38,41 & 38,74 & 39,1 & 39,61 & 40,81 & 41,26 & 42,20 & 43,03 \\
\hline Contratada (4) & & & & & & & & 41,22 & 42,12 & 43,04 \\
\hline TOTAL & 35,26 & 35,43 & 36,05 & 36,38 & 36,58 & 37,17 & 38,1 & 38,64 & 39,26 & 39,90 \\
\hline TOTAL (4) & & & & & & & & 37,99 & 38,51 & 39,09 \\
\hline
\end{tabular}

(1) La categoría de Emérita, en los cursos 11-12, 12-13 y 13-14, se incluye en el personal contratado.

(2) El total de funcionarias incluye: CU, TU, CEU, TEU y otro personal funcionario.

(3) Profesorado Contratado: Contratado Doctor, Ayudante Doctor y Contratado de universidad privada.

(4) Universidades públicas, centros propios.

Fuente: Elaboración propia a partir de la los datos del Instituto de la Mujer hasta 2010-2011. A partir del 2011-12, la fuente es la estadística del MECD: EPU [actualizado: 30/08/2015].

Por ramas de conocimiento, en las CC. Técnicas la segregación de género, con un $20,6 \%$ de mujeres, es similar a la de la etapa de formación. Sin embargo no alcanzan la presencia equilibrada en Ciencias (38,4\%), aunque sí en Humanidades $(47,6 \%)$, en CC. de la Salud y CC. Sociales y Jurídicas $(44,2 \%)^{17}$.

En resumen, en el PDI hay dos segregaciones de género: vertical, por categorías $^{18}$, y horizontal por ramas de conocimiento. Si bien ha aumentado el número de catedráticas y de titulares, en Arquitectura e Ingeniería son sólo el 10\% y 21,6\% respectivamente. Además, en CC. de la Salud, Ilama la atención, por el elevado número de alumnas, la desproporción entre CU y TU, por un lado, y contratadas por otro: mientras las primeras rozan el 20\% (CU: 19,8\%) y el 40\% (TU: 39,8\%), el porcentaje de contratadas (46,3\%) está más próximo al del resto de ramas de conocimiento, exceptuando, una vez más, ingeniería (23\%). Por último, en Arte y Humanidades es donde más mujeres hay en las tres categorías con relación a las otras ramas de conocimiento, superando las contratadas el 50\% (Figura 1).

A diferencia de lo que ocurre en el PDI, en el PAS las mujeres son el 59\%, superando el $68 \%$ del total de funcionarios (25 pp más numeroso que el de laborales) y cumpliéndose la presencia equilibrada para el caso del personal contratado (Tabla 3).

16. Primer curso sobre el que se publica esta estadística: 2004-2005 (Martín Bardera, 2015: 244).

17. Datos para PDI de centros propios de universidades públicas (MECD, 2015: 129 y 135).

18. En 2007, ninguna rama del conocimiento llegaba al 20\% de mujeres $C U$, aproximándose humanidades con el 19,6\% (Martín Bardera, 2015: 248). 
Tabla 3. PAS desagregado por sexo según la vinculación laboral. Curso 2013-2014

\begin{tabular}{|c|c|c|c|c|c|c|c|c|}
\hline \multirow{3}{*}{$\begin{array}{l}\text { CENTROS PROPIOS } \\
\text { CURSO 2013-2014 } \\
\end{array}$} & \multirow{2}{*}{\multicolumn{2}{|c|}{ TOTAL PAS }} & \multicolumn{6}{|c|}{ TIPO DE VINCULACIÓN LABORAL } \\
\hline & & & \multicolumn{2}{|c|}{ Funcionariado } & \multicolumn{2}{|c|}{ C. Indefinido } & \multicolumn{2}{|c|}{ C. Temporal } \\
\hline & $\% M$ & $\% \mathbf{H}$ & $\% M$ & $\% \mathbf{H}$ & $\% \mathbf{M}$ & $\% \mathbf{H}$ & $\% M$ & $\% \mathbf{H}$ \\
\hline TOTALES & 59,09 & 40,91 & 68,26 & 31,74 & 42,04 & 57,96 & 48,23 & 51,77 \\
\hline U. Presenciales & 58,79 & 41,21 & 67,92 & 32,08 & 41,85 & 58,15 & 48,10 & 51,90 \\
\hline UNED & 69,13 & 30,87 & 80,48 & 19,52 & 42,90 & 57,10 & 55,00 & 45,00 \\
\hline $\begin{array}{l}\text { U. Especiales (UNIA } \\
\text { y UIMP) }\end{array}$ & 61,96 & 38,04 & 61,59 & 38,41 & 63,64 & 36,36 & 0,00 & 0,00 \\
\hline
\end{tabular}

Fuente: Elaboración propia a partir de los datos del MECD: EPU, curso 2013-2014.

Tabla 4. Porcentaje de mujeres y hombres del PAS por funciones.

Curso 2013-2014

\begin{tabular}{lrrr}
\hline & TOTAL & \% Mujeres & \% Hombres \\
\hline Personal administrativo & 58,3 & 66 & 34 \\
Apoyo a la investigación & 5,6 & 50 & 50 \\
Apoyo a la educación & 6,7 & 50,2 & 49,8 \\
Mantenimiento y servicios & 8,5 & 35,1 & 64,9 \\
Apoyo sanitario y social a alumnado & 8,7 & 64,9 & 35,1 \\
Personal administrativo directivo & 4,5 & 55,2 & 44,8 \\
Otros & 7,7 & 58,1 & 41,9 \\
\hline
\end{tabular}

Fuente: Elaboración propia a partir de los datos del MECD (2015: 131, 143).

Con relación a los distintos grupos, las funcionarias superan a los funcionarios en todos, sobrepasando el 60\% en los subgrupos: A2, C1 y C2. Es destacable que en $\mathrm{C} 1$ y C2, los más numerosos en general, alcancen el 70,3\% y el 75,6\% respectivamente. Entre el personal laboral fijo, la presencia es equilibrada en todos los grupos excepto en el GIII (Bachillerato) donde las mujeres se quedan en el 37,6\% (MECD, 2015: 141-142).

Respecto a las funciones del PAS (Tabla 4) son principalmente administrativas $(62,8 \%$ incluyendo al personal administrativo y al personal administrativo directivo). En esta división por funciones, no hay presencia equilibrada en el personal de mantenimiento y servicios, administrativo, y de apoyo sanitario y social al alumnado $(35,1 \%, 66 \%$ y $64,9 \%$ de mujeres respectivamente).

\section{Una visión de conjunto}

De acuerdo con los resultados, se puede afirmar que la universidad no discrimina ni en el acceso ni en la etapa de formación, es más, reconoce a las mujeres como buenas estudiantes. Sin embargo hay un sesgo de género en la elección de las titulaciones de Ingeniería y Arquitectura y CC. de la Salud. Hay que añadir que ambas ramas agrupan grados que requieren buena nota en el expediente para el acceso, por 
lo que la diferencia parece fruto de la elección. Así, y puesto que la institución garantiza la igualdad en el procedimiento de acceso, la orientación de las preferencias individuales se puede atribuir a la influencia de la trayectoria vital y educativa anterior.

Este sesgo es el único observado durante grado y postgrado, y el primero que se traslada a la profesión de PDI: las mujeres no ocupan cátedras, titularidades o ayudantías de las titulaciones técnicas. Sin embargo, en las Ciencias Médicas, las mujeres se aglutinan en los puestos de ayudante, asociado y colaborador (contratados en general) y los hombres son mayoría entre titulares y catedráticos (funcionarios): hay una brecha entre el número de estudiantes y el de docentes que ocupan las categorías más acreditadas, añadiéndose un sesgo vertical.

Además, desde la etapa de grado hasta la defensa de la tesis doctoral, hay una disminución paulatina de mujeres que se agudiza cuando forman parte del PDI (es el conocido "gráfico de tijera"19). La pregunta es ipor qué abandonar un medio "amable"? esto es, que durante la etapa de formación (1) prevé un sistema igualitario de acceso; (2) reconoce el desempeño sin tener en cuenta el sexo; (3) ofrece posibilidades de continuidad laboral; y (4) precisamente en un área feminizada como la enseñanza ${ }^{20}$. Las mujeres en el PDI son clave para interpretar parte del problema de la desigualdad de género, pues se forman desde el grado en la misma institución donde acaban ejerciendo su profesión. Pero además, esta institución acoge a otro colectivo laboral, el PAS, cuyas funciones de apoyo son secundarias respecto a las genuinamente universitarias: aquí las mujeres tienen mayor presencia y una promoción profesional acorde con la misma. En una visión de conjunto, este hecho es el resultado de una segregación laboral más.

Comparando de forma somera ambos colectivos profesionales ${ }^{21}$, algunas diferencias abundan en la importancia de la socialización y apuntan a la persistencia de estereotipos: (1) el oficio de secretaria es uno de los primeros socialmente aceptados al ser sus funciones, de asistencia y apoyo, parecidas a las del hogar; (2) el sistema de acceso a la función pública mediante examen reproduce el modelo que las mujeres aprendieron desde las primeras etapas educativas y que continúa en la Universidad durante la etapa de grado y máster; (3) la promoción se basa en la meritocracia y es sencilla en el procedimiento: frente a la compleja carrera docente que exige publicar, dirigir, investigar en continuidad, aquí se trata de rendir sucesivos exámenes en un sistema de oposiciones que permite administrar mejor el tiempo, sin que los periodos de ausencia penalicen el currículum; y (4) las condiciones de trabajo en la administración incentivan la conciliación entre familia y empleo ${ }^{22}$.

19. La obtención de ayudas postdoctorales representan el primer punto de inflexión, descendiendo más de 10 pp respecto a las predoctorales y acabando con la presencia equilibrada: año 2012, becarias predoctorales FPI y FPU, 50,9\% y 52,7\%; postdoctorales RyC y Juan de la Cierva, 39\% y 38,3\% respectivamente (MECD, 2014). En el curso 2013, las mujeres RyC bajan al 35,9\% (MECD, 2015). Faltan datos para saber por qué (MICINN, 2011: 26-29 y 78).

20. Que educación y salud sean las áreas más feminizadas en la educación terciaria en todos los países de la UE-27, superando en 2011 el 70\%, no tiene continuidad en las categorías profesionales más altas. Fuentes: Eurostat; Martín Bardera (2015: 221-230).

21. Para un análisis más detallado, Martín Bardera (2015: 265-268).

22. La administración pública es pionera en implementar medidas a favor de la misma (MAP, 2006). Aunque se prefiere el término "corresponsabilidad", que incorpora los roles cuidador y proveedor de cual- 


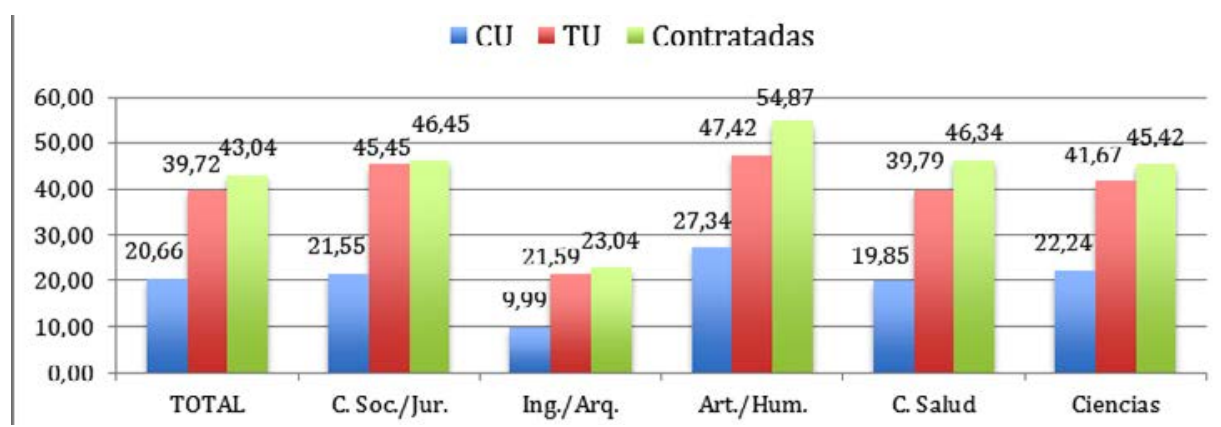

Figura 1. Mujeres PDI por categoría profesional en cada rama de conocimiento. Curso 2013-2014. Fuente: Elaboración propia a partir de los datos del MECD: EPU, curso 2013-2014.

En conclusión: hay falta de equidad en la universidad, afecta a todos los colectivos aunque no de igual manera y ello no significa siempre que la universidad, como institución, discrimine directamente.

\section{LAS UNIDADES DE IGUALDAD}

Ante esta situación cabe preguntarse, qué repercusión han tenido las políticas públicas de igualdad de género en las universidades españolas. En concreto, ¿cuentan con un modelo adecuado para fomentar dichas políticas? y iestán incorporando los cambios legislativos como parte de su actuación pública? Para responder a la primera pregunta se ha revisado si las universidades cuentan con Ul y cómo se organizan. Para responder a la segunda pregunta se han seleccionado cuatro acciones básicas: la redacción de PI y diagnósticos de situación; la elaboración de estadísticas desagregadas por sexo; la impartición de posgrados específicos y la formación de equipos rectorales respetando el criterio de presencia equilibrada ${ }^{23}$. Para sistematizar la información en las 49 universidades públicas ${ }^{24}$ se ha creado una base de datos a partir de la página web de cada institución ${ }^{25}$.

Un estudio completo incluiría líneas de investigación, asignaturas de creación específica y de grado, presupuestos anuales y reforma de los estatutos. Aspectos que se señalan por su relevancia, si bien no es posible abordar aquí ${ }^{26}$. También se debe considerar que aunque los diagnósticos de situación y los planes de igualdad reflejasen toda esta casuística, han sido elaborados en distintos momentos y un análisis pormenorizado de los mismos no es el objetivo. El planteamiento es que si las acciones propuestas son las más visibles y su coste es bajo en instituciones con personal cualificado y me-

quier individuo, aquí se utiliza "conciliación" porque las propias mujeres han asumido el diagnóstico que ve, en su incorporación al trabajo, el origen de la desatención al cuidado dentro de la familia. Esto limita sus expectativas profesionales.

23. Martín Bardera (2015; 277-309; 372-472).

24. No se incluye la Universidad Internacional Menéndez Pelayo (Martín Bardera, 2015: 278).

25. Permiten acceder a muchos contenidos y son la carta de presentación más visible.

26. A la complejidad de la institución se une la exigencia de transversalidad de la perspectiva de género. Martín Bardera (2015: 277-281). 
dios técnicos, el resultado permitirá una primera estimación sobre la implementación de las políticas de igualdad en la universidad siete años después de la $\mathrm{LOI}^{27}$ y, en consecuencia, de su compromiso con la igualdad como principio informador y valor social.

\section{Unidades de igualdad y centros de estudios feministas}

Cuando se aprueba la LOI en muchas universidades ya existían, desde los años 8090, organizaciones que agrupaban a las especialistas en el área de estudios de la mujer, el género y los feminismos ${ }^{28}$. La diferencia con las UI va a estar marcada por su origen y las funciones que asumen. Así, mientras las unidades son estructuras político-administrativas que nacen con la LOI y la LOU4 (2007) ${ }^{29}$, los centros de estudios de la mujer son de corte académico, centrados en la docencia y la investigación. A mayores, hay observatorios de género con funciones técnicas dentro de las unidades -recogida de datos, elaboración de informes y diagnósticos ${ }^{30}$ - o que constituyen las propias unidades.

En un principio algunas universidades "aprovechan" los centros de estudios para iniciar las políticas de igualdad; otras veces son éstos los que toman la iniciativa ${ }^{31}$. En ambos casos ocurre respecto a una acción concreta: elaborar el diagnóstico de situación y, quizá, el I PI ${ }^{32}$. En ocasiones se integran en las nuevas unidades miembros de estos centros de estudio ${ }^{33}$. Menos frecuente es que se formalicen relaciones de colaboración ${ }^{34}$. Estas interacciones se deben a que, cuando se aprueba la LOI, los centros de estudios feministas eran el espacio que acogía a las especialistas en temas de género. Sin embargo, siendo positivo sumar fuerzas, tienen funciones diferentes.

En los centros las actividades se encaminan hacia la organización de cursos y seminarios, la investigación y la edición de publicaciones especializadas sobre mujeres. En cambio, las UI son unidades administrativas, su cometido es implementar políticas de igualdad de género al interior de las universidades. En consecuencia, sus funciones principales son la elaboración, evaluación y seguimiento de diagnósticos de situación y PI, así como el asesoramiento dentro de la institución y la formación de los distintos colectivos. Deberían afectar a la estructura de la organización, de manera coordinada y en los distintos niveles (gender mainstreaming) ${ }^{35}$.

27. El curso de referencia es el 2012-2013.

28. Martín Bardera, 2015: anexo V.

29. LOU4, 2007: Disposición adicional duodécima: la ley obliga a crear UI, no centros de estudios de la mujer.

30. Martín Bardera, 2015: anexo VII.

31. Elizondo; Novo; Silvestre (2010).

32. Así, en la Universidad Politécnica de Cataluña, se constituye en 2004 el Grupo de Investigación de Igualdad de Oportunidades de Arquitectura, Ciencia y Tecnología (su origen data de la década de 1990) que realiza la guía para elaborar el I Plan de Igualdad; en la Rovira i Virgili, el GRÈC, creado en 1989, es el antecedente del Observatorio de Igualdad y es el que elabora el Diagnostic de situación en 2005-2006; y la Fundación Isonomía, de la Jaime I de Castellón realiza el diagnóstico. Martín Bardera, 2015: anexo IX.

33. En la Comisión de Igualdad de la Universidad de Valladolid la secretaria es la directora de la Cátedra de Estudios de Género; o en la Universidad de Jaén hay representantes de los dos seminarios permanentes de estudios sobre la mujer en la Comisión que elabora el Diagnóstico de situación (2009) y el PI (2011-2015).

34. Como sucede en la Universidad Jaume I de Castellón que mantiene un convenio marco con la Fundación Isonomía para el desarrollo de las acciones del PI y está prevista la integración del Observatorio.

35. La transversalidad de género también se concreta en la presencia equilibrada de mujeres y hombres en los órganos de gobierno, representación y selección del personal, así como en la introducción de la perspectiva de género en los planes de estudio, las normas y los presupuestos. (LOI, 2007: art. 15.) Pero 
Además, otra diferencia con relación a los centros de la mujer está en el punto de partida: mientras éstos interesan sobre todo a especialistas femeninas, las unidades deben abordar acciones que no se identifiquen sólo con las mujeres al afectar la desigualdad a la sociedad en su conjunto. Entonces, para que los beneficios reviertan en toda la comunidad se requiere la implicación de los varones. En esta línea, el principio de presencia equilibrada debería respetarse en la composición de las UI. Sin embargo, se ha convertido en práctica habitual no ya que estén dirigidas por mujeres, sino que su exiguo personal y el de las comisiones asesoras, sea básicamente femenino ${ }^{36}$.

Todo lo anterior no obsta para que el trabajo de estructuras académicas y administrativas repercuta en ambas y se aprovechen los resultados: las UI deben contar con especialistas que procedan de los centros de la mujer; y, por su parte, unidades y observatorios aportan datos que pueden ser materia de estudio e investigación. Así, la colaboración es provechosa, pero deben diferenciarse funciones, estructura y resultados.

En mayo de 2014 prácticamente todas las universidades públicas tenían entre sus estructuras UI. Eran excepción la Universidad de La Rioja y la Universidad de León en cuyas páginas web no aparecía referencia alguna ${ }^{37}$. Un caso parecido era el de la Universidad Rey Juan Carlos, que llevaba al menos seis meses con la página de la unidad de igualdad en construcción ${ }^{38}$.

\section{Estructura orgánica de las unidades de igualdad}

¿Cuál es el lugar de las UI en el organigrama de la universidad? ¿Quiénes las forman? ¿Qué recursos tienen? ¿Tratan sólo de la desigualdad de género?

Respecto a la dependencia orgánica, hay tres casos: que dependan directamente del rector, que se integren en algún vicerrectorado, o que sean un servicio. De las cuarenta y siete UI veintisiete dependen de algún vicerrectorado (Tabla 5). De éstas, en cinco media un servicio, un área o un secretariado de competencias más amplias en política social o asistencia en general ${ }^{39}$. Hay ocho unidades que dependen directamente del rector (incluidas las que tienen la figura de "Delegada del rector para la igualdad"); tres de la secretaría general; una de la gerencia; y en las ocho restantes no se especifica cuál es su inserción en la estructura universitaria. De éstas, la de Cádiz está vinculada a una dirección general; la deVigo, se configura como un servicio a la comunidad (enlazada desde la web de "Administración y Servicios"); y la Miguel Hernández es una unidad administrativa.

es función de las UI destacar estas cuestiones en los diagnósticos de situación y, cuando haya que corregirlas, proponer medidas en los planes. La implicación en las UI de personal de distintos centros y servicios, es una buena estrategia de capilarización implantada en algunas universidades (Politécnica de Cataluña, Barcelona, Autónoma de Barcelona y Valencia) como se indicará después.

36. Las comisiones asesoras o de igualdad de las universidades de A Coruña, Cádiz, Extremadura, Vigo y Salamanca se forman atendiendo al principio de presencia equilibrada. Pero en las demás, si consta quiénes las forman, siempre son más mujeres o sólo mujeres. Martín Bardera, 2015: anexo VI.

37. La Unidad de Igualdad de la Universidad de La Rioja se crea en julio de 2016 y es dirigida por una persona delegada del Rector. En junio de 2017 también la Universidad de León cuenta con una Unidad de Igualdad que pertenece al Área de Responsabilidad Social, sin embargo no se hallan datos sobre su fecha de creación, composición, estructura, etc.

38. En enero de 2015 se pone en funcionamiento el Observatorio de Igualdad de Género, que incluye la Unidad de Igualdad, creada en septiembre de 2015.

39. Universidades: Carlos III, Huelva, Málaga, Oviedo y Pablo Olavide. 
Tabla 5. Unidades de igualdad y algunas acciones básicas a partir de la LOI

\begin{tabular}{|c|c|c|c|c|c|c|c|}
\hline Universidad & $\begin{array}{l}\text { Organismo de } \\
\text { Igualdad }\end{array}$ & $\begin{array}{c}\text { Dependencia } \\
\text { orgánica }\end{array}$ & Plan & $\begin{array}{l}\text { Aprob. I } \\
\text { Plan }\end{array}$ & Vigencia & $\mathrm{E} / \mathrm{D}^{*}$ & Posgrados \\
\hline EHU & Dirección & Vicerrectorado & 1 & 2010 & 2010-2013 & Sí & $\begin{array}{l}\text {-Máster } \\
\text {-Doctorado }\end{array}$ \\
\hline UA & Unidad & $\begin{array}{l}\text { Delegación del } \\
\text { rector }\end{array}$ & II & 2010 & 2014-2016 & Sí & -Doctorado Inter.(1) \\
\hline$\cup A B$ & Observatori & Vicerrectorado & III & 2006 & $2013-2017$ & Sí & $\begin{array}{l}\text {-Máster Inter. (2) } \\
\text {-Itinerario-Grado }\end{array}$ \\
\hline $\mathrm{UAH}$ & Unidad & Secretaría General & - & & & - & -Doctorado Inter.(1) \\
\hline UAL & Secretariado & Vicerrectorado & - & & & - & -Máster \\
\hline UAM & Unidad & Vicerrectorado & I & 2011 & 2011-2014 & Sí & $\begin{array}{l}\text {-Máster } \\
\text {-Doctorado Inter. (1) }\end{array}$ \\
\hline UB & Unitat & $\begin{array}{l}\text { Delegada del } \\
\text { rector }\end{array}$ & II & 2007 & $2011-2013$ & Sí & $\begin{array}{l}\text {-Máster Inter. (2) } \\
\text {-Máster N.O (8) }\end{array}$ \\
\hline UBU & Unidad & Vicerrectorado & I & 2013 & $2013-2016$ & - & -Máster N.O. \\
\hline UC & Unidad & Vicerrectorado & 1 & 2009 & $2009-2010$ & - & -Doctorado Inter.(3) \\
\hline UC3M & Unidad & $\begin{array}{l}\text { Área dependiente } \\
\text { de un } \\
\text { Vicerrectorado }\end{array}$ & I & 2010 & 2010-2013 & Sí & \\
\hline UCA & Unidad & Dirección General & I & 2011 & 2011-2015 & Sí & -Máster Inter.(4) \\
\hline UCLM & Unidad & Vicerrectorado & - & & & Sí & $\begin{array}{l}\text {-Máster N.O. } \\
\text {-Especialista (8) }\end{array}$ \\
\hline UCM & Unidad & $\begin{array}{l}\text { Delegación del } \\
\text { rector }\end{array}$ & I & 2009 & $2009-2011$ & Sí & $\begin{array}{l}\text {-2 Máster } \\
\text {-2 Doctorado } \\
\text {-Master N.O. }\end{array}$ \\
\hline UCO & Unidad & Secretaría General & - & & & Sí & \\
\hline UDC & Oficina & Vicerrectorado & I & 2013 & $2013-2017$ & Sí & -Doctorado Inter.(3) \\
\hline UDG & Unitat & Vicerectora & 1 & 2009 & 2008-2012 & Sí & -Máster Inter.(2) \\
\hline UDL & Centre & Vicerrectorat & 1 & 2008 & $2008-2011$ & Sí & $\begin{array}{l}\text {-2 Máster } \\
\text {-Máster Inter. (2) }\end{array}$ \\
\hline UGR & Unidad & $\begin{array}{l}\text { Delegación del } \\
\text { rector }\end{array}$ & I & 2010 & 2011-2014 & Sí & $\begin{array}{l}\text {-Máster Erasmus Mundus } \\
\text {-Doctorado }\end{array}$ \\
\hline UHU & Unidad & $\begin{array}{l}\text { Servicio } \\
\text { dependiente de } \\
\text { un Vicerrectorado }\end{array}$ & 1 & 2011-2012 & $2012-2013$ & & $\begin{array}{l}\text {-Máster Inter. (4) } \\
\text {-Doctorado Inter.(1) }\end{array}$ \\
\hline UJAEN & Unidad & Vicerrectorado & 1 & 2011 & 2011-2015 & - & $\begin{array}{l}\text {-Máster N.O. } \\
\text {-Máster (5) }\end{array}$ \\
\hline UנI & Unidad & Vicerectorat & I & 2010 & 2010-2014 & - & $\begin{array}{l}\text {-Máster Inter. (6) } \\
\text {-Doctorado Inter. (1) } \\
\text {-Máster } \\
\text {-Especialista (Isonomía) }\end{array}$ \\
\hline ULE & - & - & 1 & 2010 & 2011-2013 & - & \\
\hline ULL & Unidad & & 1 & 2013 & 2014-2017 & Sí & $\begin{array}{l}\text {-Máster } \\
\text {-Máster N.O. } \\
\text {-Experto } \\
\text {-Especialista } \\
\text {-Doctorado Inter. (1) }\end{array}$ \\
\hline ULPGC & Unidad & Secretaría General & No & & & Sí & -Experto (8) \\
\hline UM & Unidad & Rector & I & 2013 & $2013-2015$ & - & $\begin{array}{l}\text {-Máster } \\
\text {-Dotorado }\end{array}$ \\
\hline UMA & Unidad & $\begin{array}{l}\text { Secretariado } \\
\text { dependiente de } \\
\text { un Vicerrectorado }\end{array}$ & II & 2010 & 2014-2018 & - & -Máster \\
\hline
\end{tabular}




\begin{tabular}{|c|c|c|c|c|c|c|c|}
\hline UMH & Unidad & $\begin{array}{l}\text { Unidad } \\
\text { administrativa }\end{array}$ & - & & & - & -Máster Inter. (6) \\
\hline UNED & Oficina & & - & & & - & -Experto \\
\hline UNEX & Oficina & Vicerrectorado & 1 & 2009 & 2009-2011 & Sí & -Especialista \\
\hline UNIA & Unidad & Vicerrectorado & - & & & & -Máster (5) \\
\hline UNIB & Oficina & Vicerrectorado & - & & & - & $\begin{array}{l}\text {-Máster } \\
\text {-Doctorado Inter. (1) }\end{array}$ \\
\hline UNIOVI & Unidad & $\begin{array}{l}\text { Servicio } \\
\text { dependiente de } \\
\text { un Vicerrectorado }\end{array}$ & I & 2013 & 2013-2015 & - & $\begin{array}{l}\text {-Master } \\
\text {-Doctorado } \\
\text {-Erasmus Mundus } \\
\text {-Doctorado Inter. (3) }\end{array}$ \\
\hline UNIZAR & Observatorio & Vicerrectorado & - & & & - & $\begin{array}{l}\text {-Máster } \\
\text {-Doctorado } \\
\text {-Máster N.O. }\end{array}$ \\
\hline UPC & $\begin{array}{l}\text {-Oficina } \\
\text {-Unitat }\end{array}$ & Vicerrectorado & II & 2007 & $2013-2015$ & Sí & -Máster Inter.(2): \\
\hline UPCT & Comisión & Rector & 1 & 2010 & $2010-2011$ & - & \\
\hline UPF & UPF Igualdad & Vicerrectorado & I & 2008 & $2008-2010$ & Sí & -Máster Inter.(2) \\
\hline UPM & Unidad & Gerencia & 1 & 2007 & 2008-2011 & - & \\
\hline UPNA & Unidad & Vicerrectorado & III & 2007 & $2007-2011$ & Sí & -Experto \\
\hline UPO & Oficina & $\begin{array}{l}\text { Unidad } \\
\text { dependiente de } \\
\text { un Vicerrectorado }\end{array}$ & 1 & 2012 & $2012-2015$ & - & -Máster \\
\hline UPV & Unidad & Vicerrectorado & 1 & 2014 & 2014-2016 & - & \\
\hline UR & - & - & - & - & - & - & -Diploma \\
\hline URJC & Unidad & & - & & & - & $\begin{array}{l}\text {-Grado (7) } \\
\text {-2 Máster } \\
\text {-Doctorado Inter. (1) }\end{array}$ \\
\hline URV & Observatori & Rector & II & 2007 & 2011-2015 & - & $\begin{array}{l}\text {-Máster Inter. (2) } \\
\text {-Módulo interdisciplinar }\end{array}$ \\
\hline US & Unidad & $\begin{array}{l}\text { Delegación del } \\
\text { rector }\end{array}$ & 1 & 2009 & 2009-2012 & Sí & -Máster \\
\hline USAL & Unidad & & 1 & 2008 & 2008-2009 & Sí & $\begin{array}{l}\text {-Máster } \\
\text {-Doctorado } \\
\text {-Máster N.O. }\end{array}$ \\
\hline USC & Oficina & Vicerrectorado & II & 2009 & 2014-2018 & Sí & $\begin{array}{l}\text {-Master } \\
\text {-Doctorado Inter. (3) }\end{array}$ \\
\hline UV & Unitat & Vicerrectorado & II & 2009 & $2013-2017$ & Sí & $\begin{array}{l}\text {-Doctorado } \\
\text {-Máster } \\
\text {-Máster }\end{array}$ \\
\hline UVA & Comisión & & 1 & 2012 & 2012-2014 & Sí & \\
\hline UVIGO & Unidade & Servicio & 1 & 2012 & $2012-2014$ & Sí & $\begin{array}{l}\text {-Máster } \\
\text {-Doctorado Inter. (3) }\end{array}$ \\
\hline
\end{tabular}

Fuente: Elaboración propia a partir de datos obtenidos de las páginas web de las universidades (Martín Bardera: 2015).

* E/D: Estadísticas desagregadas por sexo.

(1) Programa de Doctorado en Estudios Interdisciplinares de Género. Universidades: Autónoma de Madrid, Alcalá, Alicante, Huelva, La Laguna, Illes Balears, Jaume I de Castellón y Rey Juan Carlos; CSIC). Verificado por ANECA.

(2) Máster en Estudis de Dones, Gènere i Ciutadania. Universidades: Barcelona (coord.), Autònoma de Barcelona, Politècnica de Catalunya, Rovira i Virgili, Girona, Lleida, Vic y Pompeu Fabra. Impulsado por el iiEDG (Institut Interuniversitari d'Estudis de Dones i Gènere. Verificado por ANECA.

(3) Doctorado Interuniversitario en Equidad e Innovación en Educación. Universidades: Vigo (coord.), A Coruña, Cantabria, Oviedo y Santiago de Compostela. No es sobre género propiamente dicho. Pueden acceder los estudiantes que hayan cursado el Máster Universitario Educación, Xénero e Igualdade por la USC, entre otros.

(4) Máster Interuniversitario y Doctorado en Género, Identidad y Ciudadanía Universidades de Cádiz y Huelva. Verificado por ANECA.

(5) Género, Feminismos y Ciudadanía: perspectivas para un nuevo siglo. Máster pendiente de verificación ANECA. Universidad Internacional de Andalucía (coord.) y Universidad de Jaén.

(6) Máster Universitario en Igualdad y Género en el Ámbito Público y Privado: Universidad Jaume I y Universidad Miguel Hernández. Verificado por ANECA.

(7) Grado en Igualdad de Género (desde el curso 2010-11 se anula en abril de 2014).

(8) Título Propio = Máster N.O. (no oficial); Experto y Especialista. 
Respecto a quiénes forman las unidades de igualdad, básicamente, una directora, algún técnico -en el mejor de los casos- y una persona de apoyo administrativo. Veintisiete de ellas incluyen una comisión o consejo donde la representación de los sectores varía y que, como ya se ha indicado, no suele tener presencia equilibrada ${ }^{40}$. Cuando el observatorio de igualdad está integrado en la unidad, realiza funciones de tratamiento de datos y análisis de situación. Hay nueve observatorios y tres comisiones que forman parte de la estructura de igualdad, aunque no está claro en todos los casos cuál es su dependencia orgánica respecto a ésta ${ }^{41}$. En la Universidad de Salamanca sus miembros son elegidos por el claustro.

Cabe destacar que la implicación en las comisiones o consejos asesores de personal procedente de distintos centros y servicios es una buena estrategia para transversalizar las políticas de igualdad. Así por ejemplo, la Universidad Politécnica de Cataluña incluye en la Comisión de Igualdad un representante por cada centro docente (preferentemente del equipo de gobierno); la Universidad Autónoma de Barcelona introdujo en el II Plan representantes de políticas de igualdad en los centros y en los departamentos ${ }^{42}$ y las Universidades de Barcelona y Valencia tienen, además de la Comisión de Igualdad de la universidad, una por cada centro ${ }^{43}$.

En el tema de los recursos materiales, además de presupuesto, instalaciones y dotación de equipos informáticos, sería necesario que los puestos administrativos y técnicos fuesen de plantilla, pues ello contribuiría a fijar la labor de las unidades. La voluntariedad y el nombramiento de becarios no resuelven la falta de continuidad. Es más, ésta se agrava si el reemplazo de los equipos que desarrollan la actividad diaria se produce al tiempo que la dirección ${ }^{44}$. Incluso en el caso de las comisiones asesoras, sería más apropiado que sus miembros proviniesen de puestos representativos de la comunidad universitaria, con lo cual, se renovarán las personas, pero no su posición en el organigrama de la universidad. La consecuencia de la inestabilidad del personal no es tanto que se produzcan nuevas orientaciones por cambios en la dirección, sino la falta de continuidad entre períodos. La creación de una plantilla minimizaría este riesgo, contribuyendo a institucionalizar las unidades, como ocurre con los servicios, departamentos y centros universitarios ${ }^{45}$.

40. Si se aboga porque los órganos de representación y gobierno, así como las distintas comisiones, tengan composición equilibrada, sería deseable que también las comisiones directivas y asesoras de las UI la tuviesen. En este caso, y a mayores, para redundar en la idea de que la desigualdad no es un problema sólo de las mujeres, sino de toda la sociedad.

41. En la Autónoma de Barcelona y en la Rovira i Virgili los observatorios de género hacen la función de las unidades. También parece el caso de la Universidad de Zaragoza donde sólo hay observatorio. En la Universidad de las Islas Baleares el Observatorio (2004), pasa a ser la Oficina para la Igualdad (2007). La Politécnica de Catalunya es la única universidad donde la unidad forma parte de la comisión. Martín Bardera, 2015: anexos VI y VII.

42. Medida cumplida al $100 \%$ en las facultades y en un $71,9 \%$ en los departamentos (entre los que más incumplieron, los de C. de la Salud y los Tecnológicos).

43. Martín Bardera, 2015: anexo VIII.

44. Lo cual, teniendo en cuenta la dependencia orgánica de la mayor parte de las unidades, es común que pase cuando cambia el equipo de gobierno.

45. Se comparte con Elizondo; Novo; Silvestre (2010) que es una debilidad la falta de institucionalización de los organismos de igualdad universitarios. 
Otro asunto relacionado con la estructura de las UI es si sólo se ocupan de la igualdad de género o si incluyen otras causas de desigualdad ${ }^{46}$. En principio, hay que distinguir la desigualdad de género por su carácter transversal y por su origen. Tomada la diferencia como marco, hay similitudes y, por tanto, indicadores, planteamientos de justificación o deslegitimación que podrían utilizarse en distintas causas $^{47}$, siendo la economía de medios una posible razón para justificar el tratamiento común. Sin embargo, si se admite que la categoría "mujer" reúne a las mujeres como grupo susceptible de discriminación, esto añade un plus a cualquier situación de desigualdad ${ }^{48}$. A ello se une el objetivo de llegar a sociedades donde sexo y género no signifiquen nada: ni colectivamente ni para el desarrollo individual, lo que convierte en transitorios planes y medidas de discriminación positiva.

\section{ACCIONES básiCAS A PARTIR DE LA LOI}

Aquí se presentan resultados del análisis de cuatro acciones que pueden servir de indicadores para valorar la puesta en marcha de las políticas de igualdad: planes de igualdad y diagnósticos de situación, incorporación de estadísticas desagregadas por sexo, impartición de posgrados oficiales específicos (Tabla 5) y formación de equipos rectorales atendiendo al criterio de presencia equilibrada (Tabla 6).

Tabla 6. Presencia equilibrada en los equipos rectorales. Curso 2012-2013

\begin{tabular}{|c|c|c|c|c|c|c|c|}
\hline \multirow{2}{*}{ CC.AA. } & \multirow{2}{*}{ ACRÓNIMO } & \multicolumn{2}{|c|}{ GOB. $1^{\text {er }}$ NIVEL } & \multicolumn{2}{|c|}{ GOB. $2^{\circ}$ NIVEL } & \multicolumn{2}{|c|}{ TOTAL GOBIERNO } \\
\hline & & $\% \mathrm{H}$ & $\% \mathrm{M}$ & $\% \mathrm{H}$ & $\% M$ & $\% \mathrm{H}$ & $\% M$ \\
\hline \multirow{10}{*}{ ANDALUCÍA } & UAL & 72,73 & 27,27 & 66,67 & 33,33 & 68,00 & 32,00 \\
\hline & UCA & 60,00 & 40,00 & 50,00 & 50,00 & 54,17 & 45,83 \\
\hline & UCO & 80,00 & 20,00 & & & 80,00 & 20,00 \\
\hline & UGR & 50,00 & 50,00 & 68,67 & 31,33 & 65,66 & 34,34 \\
\hline & UHU & 62,50 & 37,50 & 55,56 & 44,44 & 57,69 & 42,31 \\
\hline & UJAEN & 63,64 & 36,36 & 66,67 & 33,33 & 65,96 & 34,04 \\
\hline & UMA & 54,55 & 45,45 & 75,00 & 25,00 & 67,74 & 32,26 \\
\hline & US & 37,50 & 62,50 & 67,86 & 32,14 & 56,82 & 43,18 \\
\hline & UNIA & 53,85 & 46,15 & 75,00 & 25,00 & 61,90 & 38,10 \\
\hline & UPO & 72,73 & 27,27 & 66,67 & 33,33 & 69,57 & 30,43 \\
\hline ARAGÓN & UNIZAR & 60,00 & 40,00 & 70,00 & 30,00 & 66,67 & 33,33 \\
\hline \multirow{2}{*}{ CANARIAS } & ULL & 61,54 & 38,46 & 46,67 & 53,33 & 51,16 & 48,84 \\
\hline & ULPGC & 60,00 & 40,00 & 67,74 & 32,26 & 65,85 & 34,15 \\
\hline CANTABRIA & UC & 72,73 & 27,27 & & & 72,73 & 27,27 \\
\hline C.-LA MANCHA & UCLM & 70,00 & 30,00 & 58,33 & 41,67 & 63,64 & 36,36 \\
\hline
\end{tabular}

46. Las universidades de Burgos, Barcelona, Pompeu Fabra, Politécnica de Madrid y la UNED, incluyen la atención a otros colectivos vulnerables (Martín Bardera, 2015: 289-291).

47. Se han invisibilizado colectivos, como el de personas con diversidad funcional, por no ajustarse a unos patrones de "normalidad". Desmontar esos conceptos y trasladar una nueva forma de afrontar sus necesidades y reivindicaciones a las leyes y la cultura social puede hacerse desde estrategias parecidas: inaugurando nuevas perspectivas, revisando el uso del lenguaje, realizando campañas de sensibilización, concienciando mediante formación, etc.

48. Aun siendo discutible la categoría "mujer" como colectivo o como minoría, se justifica porque incluso en las sociedades más igualitarias por ley persisten las desigualdades de género. En este sentido es significativa hasta la discriminación estadística, que asocia el hecho de ser mujer al rol social atribuido. 


\begin{tabular}{|c|c|c|c|c|c|c|c|}
\hline \multirow{4}{*}{ CASTILLA Y LEÓN } & UBU & 90,00 & 10,00 & 50,00 & 50,00 & 78,57 & 21,43 \\
\hline & ULE & 62,50 & 37,50 & \multirow[t]{3}{*}{67,74} & \multirow[t]{3}{*}{32,26} & 66,67 & 33,33 \\
\hline & USAL & 50,00 & 50,00 & & & 50,00 & 50,00 \\
\hline & UVA & 81,82 & 18,18 & & & 81,82 & 18,18 \\
\hline \multirow{7}{*}{ CATALUÑA } & UAB & 54,55 & 45,45 & & & 54,55 & 45,45 \\
\hline & UB & 64,29 & 35,71 & 50,00 & 50,00 & 59,09 & 40,91 \\
\hline & UDG & 69,23 & 30,77 & 66,67 & 33,33 & 68,75 & 31,25 \\
\hline & UDL & 75,00 & 25,00 & 55,00 & 45,00 & 62,50 & 37,50 \\
\hline & UPC & 64,29 & 35,71 & & & 64,29 & 35,71 \\
\hline & UPF & 72,73 & 27,27 & 33,33 & 66,67 & 58,82 & 41,18 \\
\hline & URV & 52,94 & 47,06 & 0,00 & 100,00 & 47,37 & 52,63 \\
\hline \multirow{6}{*}{ MADRID } & UAH & 64,29 & 35,71 & 100,00 & 0,00 & 68,75 & 31,25 \\
\hline & UAM & 46,67 & 53,33 & 60,71 & 39,29 & 55,81 & 44,19 \\
\hline & UC3M & 71,43 & 28,57 & 50,00 & 50,00 & 60,00 & 40,00 \\
\hline & UCM & 53,33 & 46,67 & 16,67 & 83,33 & 42,86 & 57,14 \\
\hline & URJC & 81,82 & 18,18 & & & 81,82 & 18,18 \\
\hline & UPM & 66,67 & 33,33 & 82,35 & 17,65 & 75,86 & 24,14 \\
\hline \multirow{5}{*}{ C. VALENCIANA } & UA & 53,85 & 46,15 & 53,33 & 46,67 & 53,57 & 46,43 \\
\hline & UV & 50,00 & 50,00 & \multirow{4}{*}{63,16} & \multirow{4}{*}{36,84} & 50,00 & 50,00 \\
\hline & UJI & 63,64 & 36,36 & & & 63,64 & 36,36 \\
\hline & UMH & 58,33 & 41,67 & & & 61,29 & 38,71 \\
\hline & UPV & 78,57 & 21,43 & & & 78,57 & 21,43 \\
\hline C. F. NAVARRA & UPNA & 80,00 & 20,00 & 55,56 & 44,44 & 68,42 & 31,58 \\
\hline EXTREMADURA & UNEX & 63,64 & 36,36 & & & 63,64 & 36,36 \\
\hline \multirow{3}{*}{ GALICIA } & UDC & 60,00 & 40,00 & 60,00 & 40,00 & 60,00 & 40,00 \\
\hline & USC & 66,67 & 33,33 & 45,45 & 54,55 & 56,52 & 43,48 \\
\hline & UVIGO & 54,55 & 45,45 & 60,00 & 40,00 & 58,54 & 41,46 \\
\hline I. BALEARES & UIB & 63,64 & 36,36 & 100,00 & 0,00 & 69,23 & 30,77 \\
\hline LA RIOJA & UR & 66,67 & 33,33 & 100,00 & 0,00 & 77,78 & 22,22 \\
\hline PAÍS VASCO & EHU & 53,85 & 46,15 & & & 53,85 & 46,15 \\
\hline P. ASTURIAS & UNIOVI & 70,00 & 30,00 & 58,33 & 41,67 & 61,76 & 38,24 \\
\hline \multirow{2}{*}{ R. MURCIA } & UM & 66,67 & 33,33 & 100,00 & 0,00 & 69,23 & 30,77 \\
\hline & UPCT & 90,91 & 9,09 & 75,00 & 25,00 & 84,21 & 15,79 \\
\hline ESTATAL & UNED & 63,64 & 36,36 & 41,18 & 58,82 & 50,00 & 50,00 \\
\hline
\end{tabular}

Resaltado: sin presencia equilibrada.

Fuente: Elaboración propia a partir de Martín Bardera (2015: anexos XII y XIII).

\section{Planes de igualdad y diagnósticos de situación}

Los PI responden al carácter estructural de la desigualdad de género al ser instrumentos para intervenir en todos los ámbitos y niveles de la organización ${ }^{49}$. Responden a la necesidad de coordinación al reunir, en un solo documento, todas las medidas definidas para un plazo temporal. Previos a los planes son los diagnósticos, que permiten conocer, a partir del análisis de datos, la realidad concreta para la que debe diseñarse el plan. De hecho, algunas veces se integran en los planes.

La LOI establece su obligatoriedad para empresas de más de 250 empleados así como para la Administración General del Estado y organismos públicos vinculados ${ }^{50}$. También el Estatuto Básico del Empleado Público lo señala ${ }^{51}$.

49. LOI (2007: art. 46).

50. LOI (2007: art. 45 y 64).

51. Ley 7/2007 de 12 de abril, del Estatuto Básico del Empleado Público, Disposición adicional octava. 
En las universidades ha sido usual que el primer diagnóstico y el primer plan los haya redactado una comisión creada al efecto, debido seguramente a la falta de un modelo administrativo para las UI. Se ha visto que la ley no explica las funciones específicas de las unidades, ni quiénes y cómo deben componerlas, tampoco orienta sobre cómo integrarlas en la organización. Se ha explicado que los centros de estudios feministas han aportado especialistas, pero el referente más próximo como modelo de organización son los observatorios. Al tener éstos un carácter eminentemente técnico, antes del desarrollo de las unidades ya se previó la necesidad de un "órgano político" que impulsara la ejecución del plan y la transversalización de sus acciones $^{52}$. Así pues, es frecuente que los primeros diagnósticos, e incluso los primeros planes, no hayan sido redactados por la unidad ${ }^{53}$.

El primer balance, al cumplirse siete años desde la aprobación de la LOI y de la Ley 7/2007, ha de tener en cuenta si las universidades cuentan con un PI, cuál es su vigencia y si ha habido otros sucesivos, incluyendo diagnósticos y/o evaluación. En mayo de 2014 doce universidades no tenían PI ${ }^{54}$; veintiocho contaban con uno, si bien la mitad con el período de vigencia cumplido; siete habían redactado el segundo, uno de los cuales venció en 2013; y dos disponían de un tercer PI, aunque uno de ellos finalizó en 2011. El balance es que, de cuarenta y nueve universidades, veintiuna estaban al día. Si se considera que los cinco planes que finalizaron en 2013 podrían estar en plazo de revisión y evaluación, serían veintiséis. Ahora bien, catorce de éstas aprobaron su primer plan entre 2010 y $2014^{55}$ (Tabla 5).

Con relación a las evaluaciones, al ser sólo nueve las universidades que han elaborado planes consecutivos, son escasas y pueden ser incluidas en el plan posterior, como ocurre en la Autónoma de Barcelona, la Politécnica de Catalunya y la Rovira i Virgili; o constituir un documento aparte, como es el caso de Málaga, Alicante, Valencia o Santiago. En las universidades de Cantabria y Sevilla, con un primer plan, se hacen informes de seguimiento. Se insiste en la importancia de esta acción ${ }^{56}$, para lo cual ayudaría fijar un procedimiento de recogida de datos, procesamiento y formulación de nuevas propuestas. Asumido el carácter estructural de la desigualdad, la continuidad es importante. Sin embargo, ante la situación descrita, no parece atrevido afirmar que dicha continuidad puede verse amenazada.

\section{Estadísticas desagregadas por sexo}

Los poderes públicos deben incluir "sistemáticamente la variable de sexo en las estadísticas, encuestas y recogida de datos" además de crear indicadores para conocer mejor la situación de mujeres y hombres y esto a fin de incorporar la perspectiva

52. "Primer plan de acción para la igualdad entre mujeres y hombres de la Universidad Autónoma de Barcelona. Bienio 2006-2007."

53. Véase Martín Bardera, 2015: 292-295; anexo X.

54. Córdoba, Islas Baleares, Las Palmas de Gran Canaria, Zaragoza, Internacional de Andalucía, Alcalá, Almería, Castilla-La Mancha, La Rioja, Miguel Hernández, UNED y Rey Juan Carlos. De las siete últimas tampoco se encuentran referencias del diagnóstico de situación.

55. 2010 (2); 2011 (3); 2012 (3); 2013 (5) y 2014 (1). Martín Bardera, 2015: anexo XI.

56. El seguimiento y la evaluación proporcionan continuidad al revisar periódicamente la implementación de los planes y el contraste con la nueva situación como previos a la redacción del siguiente plan. 
de género de manera efectiva (LOI, art.20). Una cuestión añadida es dónde se produce esa sistematización: es de esperar que en los diagnósticos de situación los datos estén desagregados por sexo, pero qué pasa con las estadísticas de la universidad. Para comprobarlo se han revisado las memorias académicas y distintos enlaces de las páginas web: "La universidad en cifras" o "Estadísticas de gestión"57. Destacan positivamente las universidades de València y de Girona, porque las páginas de sus UI recogen todas las estadísticas actualizadas, algo diferente a un informe puntual que, como se ha visto, en bastantes ocasiones no se renueva. En general, 26 universidades incluyen la variable sexo y $21 \mathrm{no}^{58}$. Se insiste en que es un mal dato, pues es una medida básica y fácil de implementar ${ }^{59}$ que hace incomprensible que el $43 \%$ de las universidades no la adopte (Tabla 5: E/D).

\section{Posgrados oficiales específicos}

La LOVG introduce la igualdad de género en la enseñanza universitaria ${ }^{60}$ en sentido amplio ${ }^{61}$. Posteriormente la LOI y la LOU4 concretarán acciones destinadas a promover cambios en la institución ${ }^{62}$. Respecto al Real Decreto 1393/2007, de ordenación de las enseñanzas universitarias oficiales, manda incorporar el derecho a la igualdad entre hombres y mujeres entre los principios que informan el diseño de los planes de estudio y en las enseñanzas relacionadas. La razón es que la enseñanza se proyecta en la actividad profesional y, cualquiera que ésta sea, debe respetarlo ${ }^{63}$. Más allá de eso, el RD 1393 no aporta nada respecto a la inclusión de asignaturas sobre igualdad de género en los grados. Se pierde la ocasión de que la materia sea común en todos los planes dentro de los 36 créditos de formación básica que fija el gobierno según ramas de conocimiento ${ }^{64}$. La incorporación de asignaturas específicas en los

57. Cursos 2011-12 y/o 2012-13 y fuentes en Martín Bardera, 2015: anexo XV.

58. En las universidades de Huelva e Internacional de Andalucía no se encuentran este tipo de documentos.

59. La información se tiene porque el MECD pide los datos desagregados por sexo.

60. "Las Universidades incluirán y fomentarán en todos los ámbitos académicos la formación, docencia e investigación en igualdad de género y no discriminación de forma transversal."

61. Abarca la formación (vinculada al aprendizaje continuo, incluiría también al PDI y al PAS), la docencia (en referencia a la enseñanza reglada) y la investigación. Ventura (2008).

62. LOI (2007: art. 25): las Administraciones públicas competentes fomentarán en el ámbito de la educación superior "la enseñanza y la investigación sobre el significado y alcance de la igualdad para mujeres y hombres" y señala tres acciones: a) incluirla en los planes de estudios; b) crear posgrados específicos; c) realizar investigaciones especializadas. También la LOU4 menciona en el preámbulo, como parte de la reforma de la ley de universidades, la creación de programas específicos sobre igualdad de género y, respecto a la investigación, además de situar la igualdad como objetivo, señala la promoción de la presencia equilibrada en los equipos de investigación (LOU4, 2007: art. 41.4).

63. RD 1393 (2007: art. 3.5.a).

64. Según Ventura (2008), los planes de estudio están incumpliendo la ley al no incorporar asignaturas o no desarrollar materias transversales de estudios de género. Para ella, desde la LOVG hasta el RD 1393, pasando por la LOI y la LOU4, se produce una dispersión normativa, pues se pasa de "incluir" (LOVG: manda) a "fomentar" (LOI: recomienda) la igualdad de género y la no discriminación. Por su parte, la LOU4 menciona los programas específicos sobre igualdad de género en los planes de estudio, algo que no desarrolla en el articulado (tampoco el RD 1393). A la dispersión normativa se suma la falta de desarrollo reglamentario. 
grados queda al libre arbitrio de las universidades y detectar esta acción excede los límites de este trabajo. Son excepciones destacables la Universitat de València ${ }^{65}$ y la Universidad Autónoma de Barcelona ${ }^{66}$.

Respecto a los postgrados especializados en temas de género e igualdad, treinta y cuatro han sido verificados por la $\mathrm{ANECA}^{67}$, de los cuales veintiséis son másteres oficiales. La Universidad Complutense es la que cuenta con más: tres másteres, tres doctorados oficiales y dos títulos propios; siete universidades no imparten ninguno ${ }^{68}$. La Universidad Politécnica de Catalunya es la única entre las politécnicas que participa en un máster interuniversitario ${ }^{69}$ (coordinado por la Universidad de Barcelona). Hay ocho universidades que no tienen postgrado oficial ni participan en interuniversitario, pero sí incluyen títulos propios $^{70}$; además, Jaén y la UNIA desarrollan un máster conjunto pendiente de verificación ANECA y las universidades de Cantabria y A Coruña solo participan en uno interuniversitario ${ }^{71}$ (Tabla 5).

En resumen, las universidades participantes en algún máster y/o doctorado oficial especializado son 31 (63\%). Se insiste en que transversalizar la igualdad de género en la formación y la docencia pasa por incorporarla a los planes de estudio del grado, en todas las ramas. Así se generaliza la idea de que "cualquier" actividad profesional debe respetar este derecho, pues a estos posgrados acceden quienes tienen un interés y un compromiso previos con las políticas de género.

\section{Presencia equilibrada en el equipo rectoral}

La presencia equilibrada es parte del proceso de empoderamiento de las mujeres y se vincula con la calidad de la democracia porque aumenta la representación en los procesos de toma de decisiones. La LOU4 confiere carácter estatutario a las normas electorales que "propicien" la presencia equilibrada en los órganos colegiados y no alude directamente a los órganos unipersonales. Sin embargo, la LOI dice que los poderes públicos "procurarán" atender a este principio en los "nombramientos y designaciones de los cargos de responsabilidad" y, además, para el caso de los centros educativos, la referencia es a los órganos de control y gobierno ${ }^{72}$. Por ello

65. Su UI detalla en la web toda la docencia de grado y posgrado sobre igualdad de género. Además, emite informes sobre la incorporación de la perspectiva de género en los programas de grado, demostrando cómo la coordinación es fundamental para una transversalización eficaz. Otras universidades que desde la UI remiten a las asignaturas sobre género: Pompeu Fabra, País Vasco, Complutense de Madrid y Girona. Cabe mencionar el único grado en Igualdad de Género en la Universidad Rey Juan Carlos (iniciado en el curso 2010-2011, fue anulado en febrero de 2014). Martín Bardera, 2015.

66. Ofrece el itinerario "Mínor d'Estudis de Gènere" (30 ECTS - 5 optativas), dirigido a estudiantes de distintos grados (se refleja en el Suplemento Europeo al Título).

67. La información obtenida desde las páginas web se ha contrastado con la información procedente del buscador de ANECA: Títulos universitarios oficiales http://srv.aneca.es/ListadoTitulos/ Junio, 13, 2106.

68. Carlos III de Madrid, Córdoba, León, Valladolid y las Politécnicas de Cartagena, Madrid y Valencia.

69. Máster en Estudis de Dones, Gènere i Ciutadania.

70. Las Palmas, Burgos, Castilla-La Mancha, Jaén, Extremadura, UNED, Pública de Navarra y La Rioja.

71. Equidad e Innovación Educativa. No es de género en sentido estricto.

72. LOI, 2007: art. 16 y art. 24.d especifican lo enunciado en el art. 14; Título V, Capítulo II sobre principio de presencia equilibrada en la AGE y en los organismos públicos (art. 52). 
los órganos colegiados y los unipersonales deben procurarla, así como los comités de selección y promoción del personal ${ }^{73}$. Ahora se trata de ver cómo se forman los equipos rectorales.

El equipo rectoral es el más visible y emblemático al representar a toda la comunidad universitaria. A su importancia se une el hecho de que, no siendo un órgano colegiado, es más fácil su constitución atendiendo a este principio. Sólo el puesto de rector es electo y al ser éste quien designa a su equipo no es preciso reformar reglamentos para que un sexo no supere el 60\%. Además, si en las categorías de titulares de universidad y escuela universitaria la presencia de mujeres está en torno al 50\%, no podrá alegarse el número para justificar su ausencia en el gobierno de la universidad. Aunque en el cuerpo de catedráticos de universidad su presencia esté en el $20 \%{ }^{74}$, sólo se requiere esta condición para ser rector. Aún así, en el curso 20122013, las universidades de Málaga y Girona eran las únicas presididas por mujeres.

En los resultados que se presentan (Tabla 6) se diferencian el primer nivel, formado por rector/a, vicerrectores, secretaria/o general y gerente ${ }^{75}$, y el segundo nivel formado por asesorías, direcciones de secretariado, vicerrectorados adjuntos, vicesecretarías generales, vicegerencias, etc. ${ }^{76}$. En el primero la distribución de cargos es más uniforme y los datos aparecen en todas las páginas web de forma clara y precisa. El criterio para considerar los cargos de segundo nivel es su carácter de puesto unipersonal y político ${ }^{77}$.

Algunos datos: En el curso 2012-2013, 17 equipos rectorales respetan el criterio de presencia equilibrada en el primer nivel ${ }^{78}$ y 15 en el segundo ${ }^{79}$. En el balance total, de las 49 universidades, 19 se ajustan a lo que marca la ley, si bien en cuatro de éstas sólo se obtuvieron datos para el primer nivel. La Universidad de Sevilla y la Pompeu Fabra son las únicas en que la diferencia de género se produce en los dos niveles -una vez a favor de los hombres y otra de las mujeres- y donde, paradójicamente, el resultado global es equilibrado. La mayor diferencia está en la Universidad Politécnica de Cartagena con el 90,9\% y el 75\% de hombres; y en Burgos donde las mujeres sólo representan el 10\% del equipo rectoral, habiendo paridad en el segundo nivel. En datos totales, los hombres superan el $70 \%$ en Politécnica de Valencia, Burgos, Politécnica de Madrid y Cantabria; y están en el 80\% o por encima, Valladolid, Rey Juan Carlos y Córdoba (Tabla 6).

73. Las Administraciones públicas deben promover la presencia equilibrada en los órganos de selección y valoración (art. 51d). Si se incumple, debe ser por "razones fundadas y objetivas, debidamente motivadas" (art. 53). Estos artículos se reproducen en la LOU 4 (2007: modificación diez, art. 13.b y modificación sesenta y cuatro, art. 62.3).

74. MECD (2015). En el curso 2012-2013 representan el $18 \%$.

75. Algunas universidades incluyen al defensor del universitario, pero aquí tiene tratamiento aparte (Martín Bardera, 2015: anexo XIV).

76. Martín Bardera, 2015: anexo XI.

77. Las jefaturas de servicio ocupadas por funcionarios de carrera, no se tienen en cuenta.

78. Las que lo incumplen tienen mayoría de hombres excepto Sevilla.

79. Para el segundo nivel se han obtenido datos de 37 universidades. Cuando no se respeta la proporción 60-40, es a favor de los hombres excepto en la Pompeu Fabra y en la Complutense (aquí las mujeres superan el $80 \%$ ). La Rovira i Virgili y Alcalá, sólo tiene dos cargos a este nivel. 
Respecto al cargo, el más feminizado es secretaria general, con 27 mujeres (55\%); y el más masculinizado (después del de rector) gerente, con sólo 10 mujeres (20\%). En los vicerrectorados, la presencia es equilibrada: de 402, 164 los ocupan mujeres (41\%). Sólo hay 9 mujeres en otros 35 cargos de este nivel, esto es un 26\% (Figura 2).

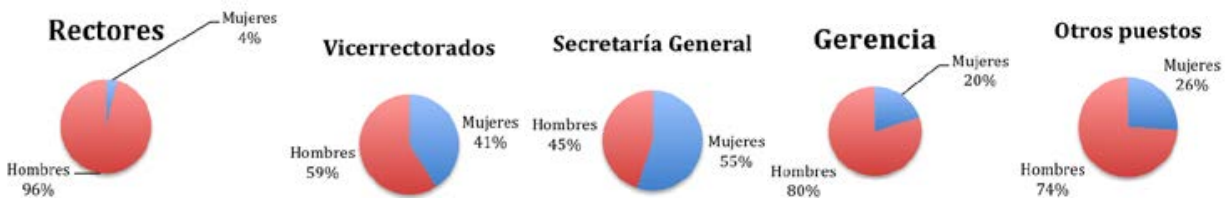

Figura 2. Mujeres en los equipos rectorales de las universidades públicas. Curso 2012-2013. Fuente: Elaboración propia a partir de Martín Bardera (2015).

Así pues, teniendo en cuenta los datos de todas las universidades públicas ${ }^{80}$, los porcentajes más equitativos por sexo se hallan en los vicerrectorados y en la secretaría general y el mayor desequilibrio en el cargo de rector. Llama la atención, por formar parte de la carrera administrativa ${ }^{81}$, que sólo un $20 \%$ de los gerentes sean mujeres.

\section{Conclusiones}

El análisis de la comunidad universitaria en femenino plural, apunta a que los sesgos de género y las desigualdades en la institución son, en buena medida, un reflejo de la desigualdad social, algo que se vincula al carácter estructural de las diferencias entre mujeres y hombres. Mérito y capacidad garantizan la igualdad en la etapa estudiantil, pero pierden eficacia con el paso del tiempo, cuando otras expectativas o responsabilidades influyen en las opciones vitales de las mujeres. En este sentido, puede aceptarse que la repercusión de la crisis en las políticas públicas ${ }^{82}$ incide en la persistencia de modelos estereotipados que se trasladan también a las universidades.

Ahora bien, hay que tener en cuenta que las universidades públicas, al formar parte de la administración del estado y ser centros de enseñanza autónomos, deben tener un papel proactivo en la consecución de la igualdad material. Por eso, en un segundo momento, se ofrecen resultados de una investigación para mostrar cómo han puesto en marcha unas pocas y sencillas acciones a las que obligan los cambios legislativos. Independientemente de la crisis económica, los resultados se atribuyen aquí más a una cuestión de voluntad en la que querer es poder.

Al cumplirse siete años de la aprobación de la LOI, todas las universidades excepto tres tenían unidad de igualdad. Sin embargo, la indefinición legal respecto a sus funciones específicas, composición y dependencia orgánica, ha contribuido a una falta de institucionalización que pone en riesgo su efectividad en la implementación de la transversalidad de género. En este sentido, la elaboración de diagnósticos y

80. Martín Bardera 2015: anexos XII y XIII.

81. Se ha visto que está muy feminizada (Tabla 2 y lo que abunda en la falta de empoderamiento.

82. Alonso (2015); Lombardo y León (2014); Paleo y Alonso (2014). 
planes de igualdad ha sido la única tarea claramente encomendada a las unidades y el $75 \%$ de las universidades habían redactado en ese momento algo parecido a un primer plan. Este porcentaje se relativiza por la falta de actualización: de los veintiún planes vigentes, catorce son posteriores a 2009 (aprobados entre 2010-2014). Con relación a las estadísticas desagregadas por sexo, el 53\% de las universidades las visibiliza. Respecto a la impartición de postgrados específicos, el 63\% de las universidades coordinaban o participaban en alguno de los 34 verificados por la ANECA. Y, por último, sólo 17 equipos rectorales (34\%) se formaron atendiendo al principio de presencia equilibrada. Además, a nivel nacional se acusa un déficit de representación femenina muy elevado entre rectores y gerentes, siendo la proporción de vicerrectoras y secretarias generales más acorde con la de profesoras.

En definitiva, los datos indican un bajo nivel de compromiso de las universidades con la igualdad efectiva. La redacción de diagnósticos de situación y planes de igualdad no es suficiente si no hay seguimiento. Además, al ser ésta una de las funciones clave de las unidades de igualdad, realizarla mal implica cuestionar éstas. Respecto a las estadísticas, no hay explicación cuando no están desagregadas puesto que el MECD las exige así. El dato sobre los posgrados parece menos negativo, si bien habría que verlo en el contexto de los estudios de grado, en atención al mandato legal de que cualquier actividad profesional incorpore este derecho fundamental. Por último, la formación de los equipos rectorales sin respetar la presencia equilibrada demuestra que la noción de empoderamiento no tiene calado. La gravedad es mayor puesto que las universidades cuentan con suficiente número de mujeres altamente cualificadas. La pregunta aquí es hasta dónde siguen existiendo los techos de cristal o en qué medida las propias estructuras y redes que sustentan el poder no han cambiado.

Para terminar, se sintetizan algunas propuestas de mejora con relación a las UI:

- Convertir a las UI en estructuras similares a los servicios universitarios, contribuiría a su institucionalización y a la consecuente continuidad de sus proyectos y actuaciones. Ello implicaría:

- Dotarlas de puestos administrativos y técnicos de plantilla

- Asignarles un crédito propio dentro del presupuesto anual de la universidad

- Adjudicarles espacio e infraestructuras

- Crear comisiones interfacultades, facilitaría la transversalización de las políticas de igualdad de género.

- Formar las comisiones vinculadas a las UI con representantes de los equipos de gobierno de facultades y centros, proporcionaría estabilidad institucional y transversalidad.

- Asumir como función prioritaria de las UI la realización de informes de seguimiento y evaluación de los PI, de modo que se sucedan los planes y actuaciones, corregiría algo en lo que parecen estar fallando muchas universidades.

En resumen, todas estas medidas fortalecerían institucionalmente a las UI, aportando continuidad y transversalidad a sus actuaciones y repercutiendo en las acciones básicas analizadas en el apartado 4. 


\section{REFERENCIAS BIBLIOGRÁFICAS}

Astelarra, J. (2005). Veinte años de políticas de igualdad. Madrid: Ediciones Cátedra. Alfama, E. y Alonso, A. (2015). Las políticas de género en la administración pública. Una introducción. Revista Vasca de Gestión de Personas y Organizaciones Públicas, 8, 24-41.

Alonso, A. (2015). Las políticas de género en España: retrocesos y resistencias en tiempos de austeridad. Ex aequo, 32, 33-48.

Instituto Nacional de Estadística. (2011). Estadística de la Enseñanza Universitaria en España. Curso 2010-2011. Recuperado de: http://www.ine.es/jaxi/menu. do?type $=$ pcaxis $\&$ path $=/$ t13/p405/a2010-2011 \&file=pcaxis.

Instituto Nacional de Estadística. (2012). Cifras INE. Boletín Informativo del Instituto Nacional de Estadística, 8. Recuperado de: http://www.inmujer.gob.es/estadisticas/ consulta.do?area=3.

Elizondo, A., Novo, A. y Silvestre, M. (2010). Políticas de igualdad en las universidades españolas. En Asociación Española de Ciencia Política, Actas del IX Congreso Español de Ciencia Política y de la Administración, Málaga: septiembre 2009. Recuperado de: http://www.aecpa.es/uploads/files/congresos/congreso_09/grupostrabajo/area01/GT04/05.pdf.

Instituto de la Mujer. http://www.inmujer.gob.es/.

Ley 7/2007, de 12 de abril del Estatuto Básico del Empleado Público. BOE núm. 89, de 13 de abril de 2007, 16270-16299.

Ley Orgánica 1/2004, de 28 de diciembre de Medidas de protección integral contra la violencia de género. BOE núm. 313, de 29 de diciembre de 2004, 42166-42197.

Ley Orgánica 3/2007, de 22 de marzo, para la igualdad efectiva de mujeres y hombres. BOE núm. 71, de 23 de marzo de 2007, 12611-12645.

Ley Orgánica 4/2007, de 12 de abril, por la que se modifica la Ley Orgánica 6/2001, de 21 de diciembre, de Universidades. BOE núm. 89, de 13 de abril de 2007, 16241-16260.

Lombardo, E. y León M. (2014). Políticas de igualdad de género y sociales en España: origen, desarrollo y desmantelamiento en un contexto de crisis económica. Investigaciones Feministas, 5, 13-35.

Martín Bardera, S. (2015). Concepto de género: de las teorías feministas a las políticas públicas. La universidad pública española como estudio de caso. Tesis doctoral dirigida por $\mathrm{M}^{\mathrm{a}}$ Teresa López de la Vieja. Universidad de Salamanca.

Martín Bardera, S. (2016). Una mirada, distintas propuestas: género y políticas públicas. Investigaciones feministas, 7(1), 289-311. Recuperado de: http://revistas. ucm.es/index.php/INFE/article/download/51954/48754.

Martínez, E. (2014). Las formas de acción colectiva: partidos políticos y movimientos sociales. En, M. Lois y A. Alonso (Coords), Ciencia política con perspectiva de género (pp. 129-159). Madrid: Akal.

Ministerio de Administraciones Públicas. (2006). Plan Concilia. Horarios y teletrabajo (Libro electrónico). Recuperado de: http://www.novagob.org/file/download/54214. 
Ministerio de Ciencia e Innovación. (2011). Libro Blanco. Situación de las Mujeres en la Ciencia Española. Madrid: Ministerio de Ciencia e Innovación. Periodo analizado: 1998-2007.

Ministerio de Ciencia e Innovación. (2012). "Subprograma Personal Técnico de Apoyo (PTA) 2012". Recuperado de: http://www.idi.mineco.gob.es/portal/site/ MICINN/menuitem.dbc68b34d11 ccbd5d52ffeb801432ea0/?vgnextoid=3ae469d ad3f88310VgnVCM1000001d04140aRCRD.

Ministerio de Educación, Cultura y Deporte. (2012). Datos y cifras del Sistema Universitario Español. Curso 2012/2013.

Ministerio de Educación, Cultura y Deporte. (2014). Datos y cifras del Sistema Universitario Español. Curso 2013-2014.

Ministerio de Educación, Cultura y Deporte. (2015). Datos y cifras del Sistema Universitario Español. Curso 2014-2015. Recuperados de http://www.mecd.gob. es/educacion-mecd/areas-educacion/universidades/estadisticas-informes/datoscifras.html.

Ministerio de Educación, Cultura y Deporte. Estadística de personal de las universidades. Curso 2013-2014. Recuperado de: http://www.mecd.gob.es/educacion-mecd/areaseducacion/universidades/estadisticas-informes/estadisticas/personal-universitario/ personal-universitario-13-14.html.

Paleo, N. y Alonso, A. (2015). ¿Es solo una cuestión de austeridad? Crisis económica y políticas de género en España. Investigaciones Feministas, 5, 36-68.

Real Decreto 1393/2007, de 29 de octubre, por el que se establece la ordenación de las enseñanzas universitarias oficiales. BOE núm. 260, de 30 de octubre de 2007, 44037-44048.

Ventura, A. (diciembre, 2008). Normativa sobre los estudios de género y universidad. Feminismo/s, 12, 155-184. 